\title{
Involvement of ribosomal protein L11 expression in sensitivity of gastric cancer against 5-FU
}

\author{
TAKUTO KAWAHATA $^{1,2^{*}}$, KOHICHI KAWAHARA ${ }^{1 *}$, MICHIKO SHIMOKAWA $^{1}$, AKIE SAKIYAMA ${ }^{1}$, \\ TAKEHIRO SHIRAISHI $^{1,2}$, KENTARO MINAMI ${ }^{1}$, MASATATSU YAMAMOTO ${ }^{1}$, YOSHINARI SHINSATO ${ }^{1}$, \\ KAZUNARI ARIMA ${ }^{2}$, TOSHIYUKI HAMADA ${ }^{2}$ and TATSUHIKO FURUKAWA ${ }^{1}$ \\ ${ }^{1}$ Department of Molecular Oncology, Kagoshima University Graduate School of Medical and Dental Sciences, Kagoshima \\ 890-8544; ${ }^{2}$ Department of Science and Engineering, Kagoshima University Graduate School of Science and \\ Engineering, Kagoshima 890-8580, Japan
}

Received January 28, 2019; Accepted June 12, 2019

DOI: $10.3892 / \mathrm{ol} .2020 .11352$

\begin{abstract}
Fluorouracil (5-FU) is widely used in the treatment of various types of solid cancer. Our study showed that ribosomal protein L11 (RPL11) was a crucial factor affecting sensitivity of gastric cancer to 5-FU, implying that RPL11 expression is a potential biomarker for predicting 5-FU sensitivity. Kaplan-Meier survival analysis indicated that high RPL11 expression in gastric cancer patients treated with 5-FU was significantly associated with good prognosis. It was therefore investigated whether RPL11 affected the sensitivity of gastric cancer against 5-FU using four human gastric cancer cell lines, MKN45 (wild-type TP53 gene), NUGC4 (wild-type), MKN7 (mutated), and KE39 cells (mutated). In vitro assays demonstrated that RPL11 knockdown in gastric cancer cell lines carrying the TP53 wild-type gene attenuated 5-FU-induced cell growth suppression and activation of the P53 pathway, but not in cells carrying mutated TP53, suggesting that 5-FU suppresses tumor progression via RPL11-mediated activation of the P53 pathway in gastric cancer. The present study provides a potential therapeutic strategy for improving 5-FU resistance in gastric cancer by elevating RPL11 expression.
\end{abstract}

Correspondence to: Dr Kohichi Kawahara or Dr Tatsuhiko Furukawa, Department of Molecular Oncology, Kagoshima University Graduate School of Medical and Dental Sciences, 8-35-1 Sakuragaoka, Kagoshima 890-8544, Japan

E-mail: k-kawahr@m3.kufm.kagoshima-u.ac.jp

E-mail: furukawa@m3.kufm.kagoshima-u.ac.jp

${ }^{*}$ Contributed equally

Abbreviations: 5-FU, 5-Fluorouracil; TP53; tumor protein p53; RPL11, ribosomal protein L11; rRNA, ribosomal RNA; MDM2, murine double minute 2; FAS, Fas cell surface death receptor

Key words: 5-Fluorouracil, gastric cancer, drug sensitivity, RPL11, $\mathrm{P} 53$ pathway

\section{Introduction}

The antimetabolite agent 5-fluorouracil (5-FU) is widely used in the treatment of many cancer types, including gastrointestinal cancers, breast cancers, lung cancers, and cancers of the aerodigestive tract (1).

5-FU inhibits DNA synthesis and RNA processing, which in turn affects cell proliferation and survival.5-FU is converted to 5-fluorodeoxyuridine monophosphate (FdUMP) in cells. FdUMP binds to the nucleotide-binding site of thymidylate synthase (TS), an enzyme that catalyzes the reaction from deoxyuridine monophosphate (dUMP) to deoxythymidine monophosphate (dTMP), and inhibits its enzymatic activity. As a result, it causes depletion or imbalance of the intracellular deoxynucleotide pool (2). FdUMP is also converted into 5-fluorodeoxyuridine triphosphate (FdUTP), which itself is a substrate for DNA polymerase and is readily misincorporated into DNA (3). Consequently, 5-FU inhibits DNA synthesis and repair and results in DNA damage (1). 5-FU can also be converted to 5-fluorouridine triphosphate (FUTP), which misincorporates into RNA molecules, particularly ribosomal RNA (rRNA), and leads to inhibition of rRNA processing (4-6). As a result, 5-FU suppresses cell proliferation.

It has been shown that nucleolar/ribosomal biogenesis stress, such as inhibition of rRNA synthesis, processing, or ribosome subunit assembly, activates tumor suppressor P53 via ribosomal proteins (RPs), including RPL5, RPL11, and RPL23 (7-13). In response to this stress, the nucleolus is disrupted, and RPL5, RPL11, and RPL23 are consequently released from the nucleolus to the nucleoplasm where they bind to and suppress MDM2, an E3 ubiquitin ligase that ubiquitinates and targets P53 for proteasome-mediated degradation, and causes P53 stabilization and activation, resulting in cell growth suppression (7-13). Recently, 5-FU has been reported to suppress tumor growth through RPL11-mediated activation of P53 in osteosarcoma (14). However, it is unknown whether 5-FU inhibits tumor growth through RPL11-mediated activation of P53 in gastric cancer.

P53 is known to play crucial roles in monitoring genomic stability and preventing malignant transformation. Activation of P53 leads to cell cycle arrest, apoptosis, or senescence, thereby preventing tumorigenesis (15). 
It has been reported that 5-FU induced wild-type P53 expression and accumulation, followed by cell growth inhibition in human gastric, human esophageal adenocarcinoma, and human embryonic fibroblast cell lines $(16,17)$. In addition, Osaki et al have reported that 5-FU induced apoptosis in gastric cancer cell lines carrying the TP53 wild-type gene (18).

Gastric cancer is the fourth most common malignancy and the second most common cause of death of all malignancies worldwide $(19,20)$. Despite declining trends globally, prevention of gastric cancer remains a priority in healthcare. Therefore, identification of potential novel factors affecting drug sensitivity of gastric cancer and preventing tumor progression is a significant clinical challenge.

In the present study, we investigated effects of RPL11 expression on the sensitivity of gastric cancer against 5-FU treatment and its underlying mechanism. Our results provide a relationship between RPL11 expression and susceptibility to $5-\mathrm{FU}$ in gastric cancer.

\section{Materials and methods}

Cell culture and reagents. Four human gastric cancer cell lines were used in this study: MKN45, NUGC4, MKN7 (all three cell lines from JCRB Cell Bank), and KE39 (from RIKEN Cell Bank). All cell lines were cultured in RPMI-1640 medium (Nissui Pharmaceutical Co., Ltd.) supplemented with $10 \%$ fetal bovine serum and $100 \mathrm{U} / \mathrm{ml}$ penicillin at $37^{\circ} \mathrm{C}$ in a humidified atmosphere of $5 \% \mathrm{CO}_{2} .5$-FU was purchased from FUJIFILM Wako Pure Chemical Corporation. 3-(4,5-Dimethyl-2-thiazolyl)-2,5-diphenyltetrazolium Bromide Thiazolyl Blue (MTT) was purchased from Nakalai Tesque.

RNA interference. Cell transfection was performed using Lipofectamine RNAiMAX Transfection Reagent (Life Technologies; Thermo Fisher Scientific, Inc.) according to the manufacturer's protocol for knockdown experiments. All siRNAs were purchased from FASMAC. siRNA sequences were as follows: siRPL11\#1, 5'-GGUGCGGGAGUAUGAGUUA-3'; siRPL11\#2, 5'-AAGGUGCGGGAGUAUGAGUUA-3'; siControl, 5'-UUCUCCGAACGUGUCACGU-3'. siP53, 5'-CGGCGC ACAGAGGAAGAGAAT-3 [Knockdown of siRNA-mediated P53 expression is previously described $(21,22)]$.

MTT assay. Cells were seeded at 7,000 cells per well in a 96-well plate and transfected with the indicated siRNAs for $24 \mathrm{~h}$. After transfection, the cells were exposed to different concentrations of 5-FU for 3 days. Subsequently, the MTT solution was added to each well, and the cells were cultured for an additional $4 \mathrm{~h}$. After removing the media, $100 \mu \mathrm{l}$ of DMSO was added to each well to dissolve the formazan crystals. The absorbance values at 570A of each well were measured with a microplate reader (Sunrise Remote; Tecan Japan Co. Ltd.) and applied to the following calculation: Relative cell viability $=\mathrm{A}$ value of cells treated with drugs/A value of cells treated with vehicle.

Immunoblot assay. For protein analysis, cells were washed twice with phosphate-buffered saline (PBS) and lysed with lysis buffer (20 mM Tris-HCl, pH 7.5, $150 \mathrm{mM} \mathrm{NaCl}, 1 \mathrm{mM}$ Sodium Vanadate, 1 mM EDTA, 50 mM NaF, 1\% Triton X-100) supplemented with Protease Inhibitor Cocktail (Nakalai
Tesque), followed by sonication to reduce viscosity. Protein concentration of each sample was determined by the Protein Assay CBB Solution, according to the manufacturer's protocol (Nakalai Tesque). Lysates containing proteins $(20 \mu \mathrm{g})$ were resolved on an SDS-polyacrylamide gel and transferred to an Immobilon-P membrane (Millipore). The membranes were blocked with blocking solution (4\% BSA in TBST; $50 \mathrm{mM}$ Tris- $\mathrm{HCl}, \mathrm{pH} 7.4,0.15 \mathrm{M} \mathrm{NaCl}$, and $0.1 \%$ Tween-20) for $1 \mathrm{~h}$ at $37^{\circ} \mathrm{C}$ prior to subsequent incubation with the following primary antibodies: Anti-P53 antibody (1:500 dilution; Santa Cruz Biotechnology), anti-P21 antibody (1:400 dilution; Santa Cruz), anti-RPL11 antibody (1:1,000 dilution; Invitrogen), and anti-Actin (1:3,000 dilution; Bio Matrix Research) primary antibodies for $1 \mathrm{~h}$ at $37^{\circ} \mathrm{C}$. Following this, the membranes were washed three times for $10 \mathrm{~min}$ in TBST and incubated with horseradish peroxidase conjugated anti-rabbit or anti-mouse IgG secondary antibodies (1:3,000 dilution; Cell Signaling Technology) for $1 \mathrm{~h}$ at $37^{\circ} \mathrm{C}$. Immunoreactive bands were visualized with enhanced chemiluminescence using Clarity Western ECL Substrate (Bio-Rad). Representative images from repeated experiments are presented in each figure.

Quantitative real-time PCR. Total RNA from cultured cells was isolated using the TRIzol reagent (Molecular Research Center) according to the manufacturer's instructions. RNA (1 $\mu \mathrm{g})$ was reverse-transcribed using the ReverTra Ace kit (Toyobo). The mRNA expression levels of P21, FAS, and RPL11 were determined by real-time RT-PCR (StepOnePlus Real-Time PCR System; Applied Biosystems) using GoTaq qPCR Master Mix (Promega) according to the manufacturer's instructions. Human GAPDH was used for normalization. The expression of the target gene was quantified using the comparative cycle threshold method. The primer sequences used were as follows: RPL11 forward, 5'-GAAAAGGAGAACCCCATGC-3' and reverse, 5'-CATTTCTCCGGATGCCAA-3'; $P 21$ forward, 5'-CTGGACTGTTTTCTCTCGGCTC-3' and reverse, 5'-TGT ATATTCAGCATTGTGGGAGGA-3'; FAS forward, 5'-TCT GCCATAAGCCCTGT-3' and reverse, 5'-GTCTGTGTACTC CTTCCCT-3'; GAPDH forward, 5'-TGCACCACCAACTGC TTAG-3' and reverse, 5'-GAGGCAGGGATGATGTTC-3'.

Kaplan-Meier plotter. The prognostic significance of the mRNA expression of RPL11 genes in gastric cancer was evaluated using the Kaplan-Meier plotter online database, including gene expression data and clinical data (GSE14210, GSE15459, GSE22377, GSE29272, GSE51105 and GSE62254). The database automatically divided RPL11 mRNA expression into high and low expression groups. The survival curve, log-rank P-value, and hazard ratio with $95 \%$ confidence intervals were calculated and displayed in the results by the computer. The datasets analyzed during the present study are available in the Kaplan-Meier plotter online database (http://kmplot. com/analysis/index.php? $\mathrm{p}=$ service \& cancer $=$ gastric).

Statistical analysis. Data are presented as the mean \pm standard deviation. Differences between multiple groups were determined by one-way analysis of variance followed by Dunnett's post-hoc test. The analyses were performed using GraphPad Prism software (version 8.1.1; GraphPad software Inc.). $\mathrm{P}<0.05$ was considered to indicate a statistically significant difference. 
A

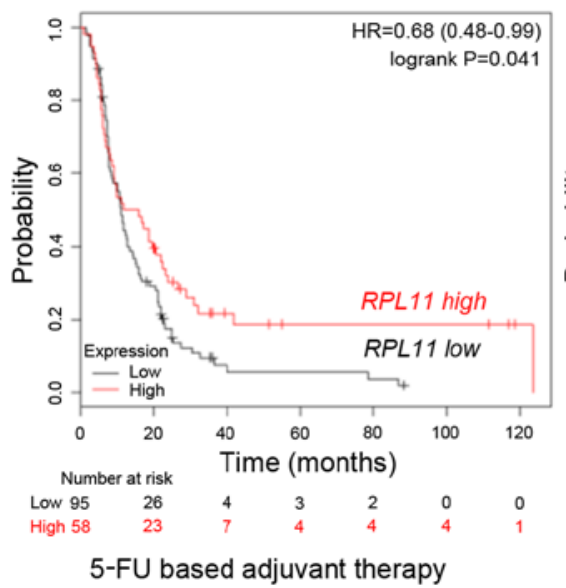

B

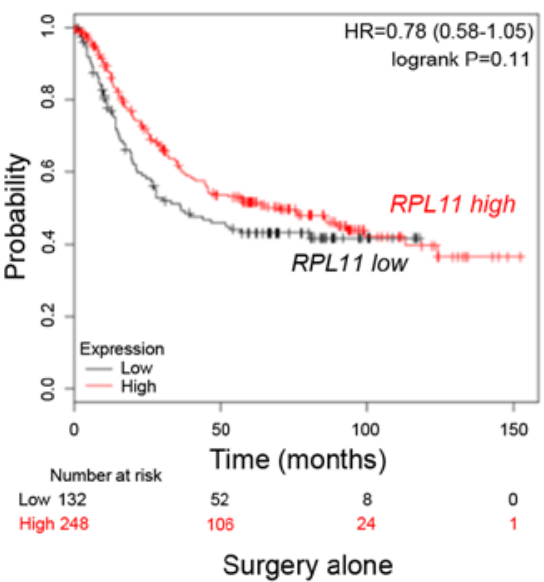

C

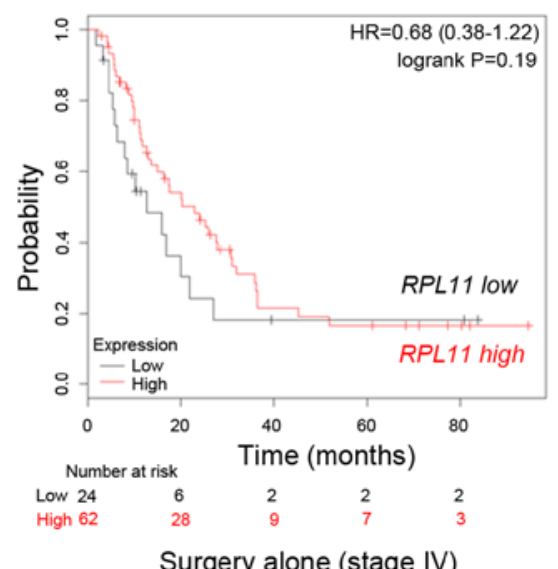

Figure 1. Kaplan-Meier analyses of associations between RPL11 expression level and overall survival of patients with gastric cancer. (A) Kaplan-Meier analyses of associations between RPL11 expression level and overall survival of gastric cancer patients treated with 5-FU based adjuvant therapy (the log-rank test was used to calculate P-values). $\mathrm{P}=0.041$ low vs. high $R P L 11$ expression group. (B) Kaplan-Meier analyses of associations between $R P L 11$ expression level and overall survival of gastric cancer patients treated by surgery alone (the log-rank test was used to calculate P-values). $\mathrm{P}=$ nonsignificant. (C) Kaplan-Meier analyses of associations between RPL11 expression level and overall survival of stage IV gastric cancer patients with surgery alone (the log-rank test was used to calculate $\mathrm{P}$-values). $\mathrm{P}=$ nonsignificant. $R P L 11$, ribosomal protein L11; 5-FU, 5-fluorouracil.

\section{Results}

RPL11 is associated with overall survival of gastric cancer patients treated with 5-FU based adjuvant therapy. To study the relationship between $R P L 11$ expression and the effect of 5 -FU on gastric cancers, we first performed survival analysis using a Kaplan-Meier plotter (www.kmplot.com). Fig. 1A indicates that 5-FU based adjuvant therapy-treated gastric cancer patients in the RPL11-high expression group showed better prognosis than those in $R P L 11$-low expression group $(\mathrm{P}=0.041)$. In contrast, gastric cancer patients treated by surgery alone showed no significant difference between the RPL11-high and RPL11-low expression groups ( $\mathrm{P}=0.11$; Fig. 1B). Similarly, survival analysis in only stage IV gastric cancer patients with surgery alone showed no significant difference between the RPL11-high and RPL11-low expression groups $(\mathrm{P}=0.19$; Fig. 1C). The results suggest that RPL11 expression alters the sensitivity of gastric cancer against 5-FU.

RPL11 inhibits cell proliferation through regulation of P53 in gastric cancer cell lines. RPL11 is known to regulate the P53 pathway (7). To investigate whether the growth inhibitory effect of 5-FU in gastric cancer involves regulation of P53 via RPL11, we performed an MTT assay using gastric cancer cell lines, MKN45 (wild-type TP53), NUGC4 (wild-type), MKN7 (mutated), and KE39 (mutated) cells. siRPL11-transfected TP53 wild-type cells, MKN45 and NUGC4 cells, showed more resistant to 5 -FU than their corresponding non-specific control siRNA-transfected cells. Further, in TP53-mutant cell lines, MKN7 and KE39 cells, there was no significant difference in growth suppressive effect of 5-FU between siRPL11-transfected cells and non-specific control siRNA-transfected cells (Fig. 2A), Further, MKN45 and NUGC4 cells with siRNA-mediated P53 knockdown showed more resistant to 5-FU than those with scramble siRNA as a control (Fig. 2B); however, double knockdown of P53 and RPL11 did not show further resistance to 5-FU in MKN45 and
NUGC4 cells in comparison with either P53 or RPL11 single knockdown (Fig. 2B). These observations suggested that cell growth suppression by RPL11-mediated P53 activation in 5-FU treated gastric cancer cell lines is dependent on normal P53 function.

5-FU treatment induces RPL11-mediated P53 activation in gastric cancer cell lines. To investigate whether RPL11 is involved in regulation of the P53 pathway in 5-FU treated gastric cancer cells, we examined protein expression levels of P53 and a P53 downstream target, P21. As a result, 5-FU increased protein expression levels of P53 and P21 in MKN45 and NUGC4 cells, and they were markedly reduced by siRNA knockdown of RPL11. Conversely, MKN7 and KE39 cells did not have any significant effects on P53 and P21 protein expression (Fig. 3A). Because the extrinsic apoptosis factor FAS is crucial for P53 mediation of apoptosis in 5-FU treated cancer cells (23), 5-FU treatment increased mRNA levels of P53 target genes, $P 21$ and $F A S$, which were determined using the quantitative real-time PCR assay. In MKN45 and NUGC4 cells, knockdown of RPL11 markedly reduced the increased mRNA levels of $P 21$ and FAS by 5-FU treatment. However, in MKN7 and KE39 cells treated with 5-FU, there was little difference in mRNA levels of $P 21$ and $F A S$ between siRPL11-transfected cell lines and non-specific control siRNA-transfected cell lines (Fig. 3B). These results demonstrate that RPL11 expression is involved in regulating the P53 pathway in 5-FU-treated gastric cancer cell lines.

\section{Discussion}

The present study investigated whether RPL11 expression was associated with the drug sensitivity of gastric cancer upon 5-FU treatment. The findings showed that high RPL11 expression in 5-FU treated gastric cancer patients have better prognosis than low RPL11 expression in 5-FU treated gastric cancer patients. In 5-FU-treated gastric cancer cell lines 

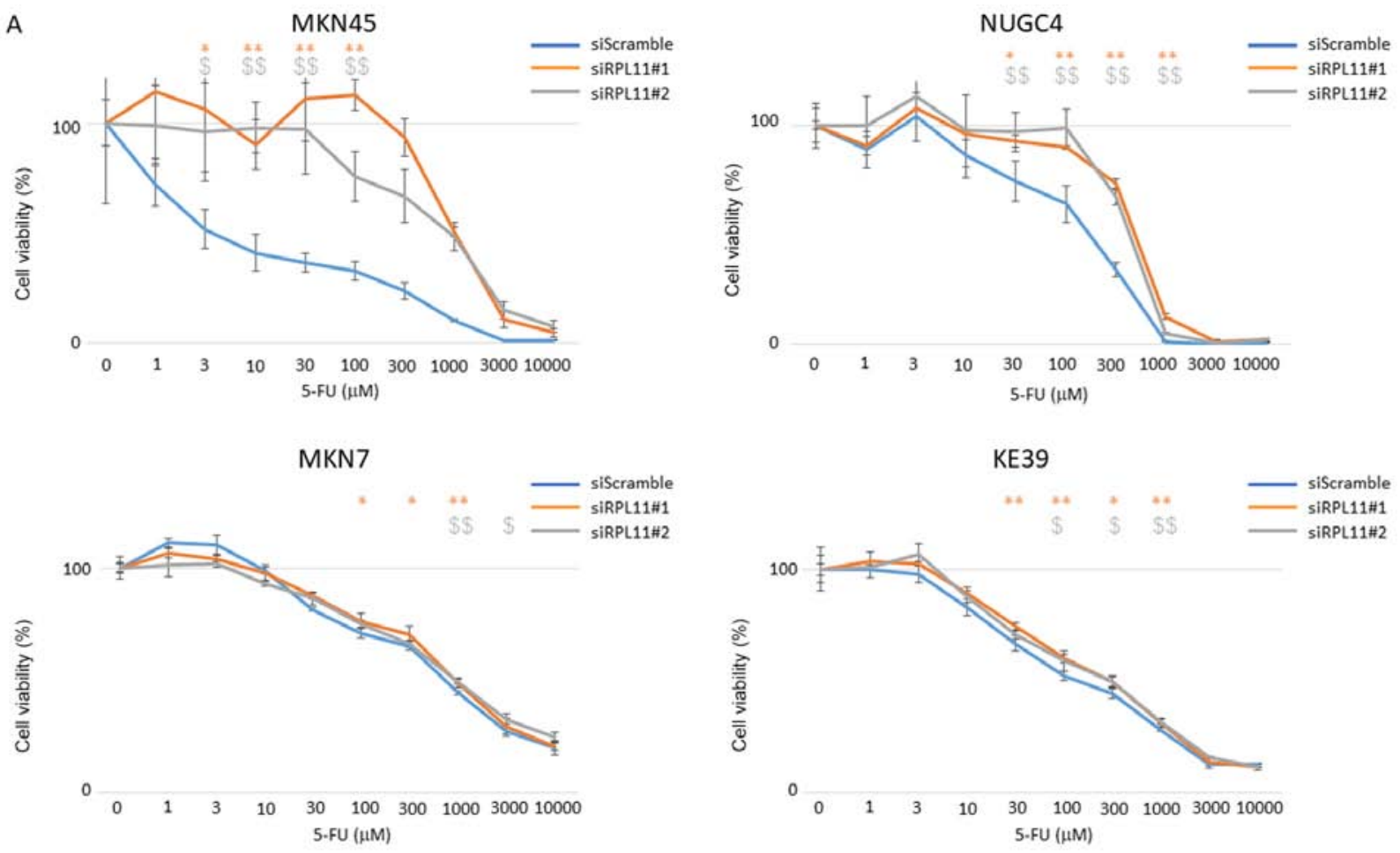

B

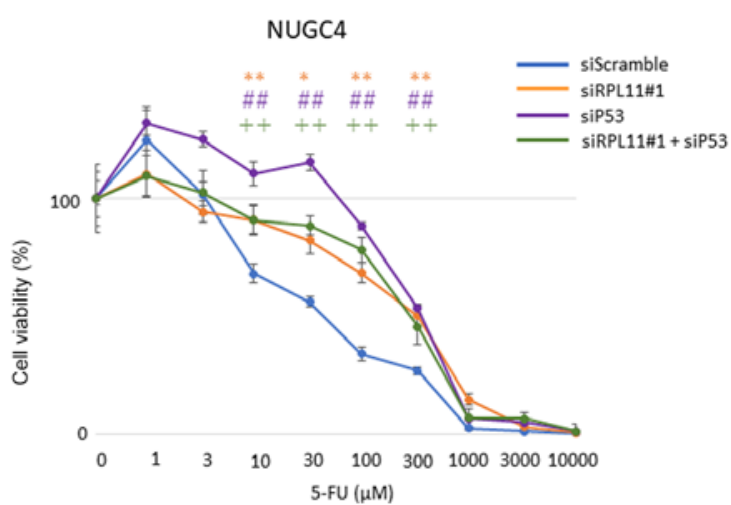

Figure 2. RPL11 is involved in viability of TP53 wild-type gastric cancer cells treated with 5-FU. (A) Viabilities of MKN45, NUGC4, MKN7 and KE39 cells transfected with siScramble (SC), siRPL11\#1, or siRPL11\#2 were analyzed by MTT assay. Cell viabilities were measured upon exposure to the step-up concentration of 5-FU for $72 \mathrm{~h}$. (B) Viabilities of MKN45, and NUGC4 cells transfected with either siScramble, siRPL11\#1, or siP53, both siRPL11\#1 and siP53 were analyzed by MTT assay. Cell viabilities were measured upon exposure to the step-up concentration of 5-FU for $72 \mathrm{~h}$. Error bars indicate the standard deviation. ${ }^{*} \mathrm{P}<0.05$ and ${ }^{* *} \mathrm{P}<0.01$ vs. siRPL11\#1 group; ${ }^{\mathrm{S}} \mathrm{P}<0.05$ and ${ }^{\$ \$} \mathrm{P}<0.01$ vs. $\mathrm{R} P L 11 \# 2$ group. ${ }^{\#} \mathrm{P}<0.05$ and ${ }^{\# \#} \mathrm{P}<0.01$ vs. siP53 group, ${ }^{+} \mathrm{P}<0.05$ and ${ }^{++} \mathrm{P}<0.01$ vs. siRPL11\#1 plus siP53 group. RPL11, ribosomal protein L11; TP53, Tumor protein p53; 5-FU, 5-fluorouracil; si, small interfering RNA.

carrying the TP53 wild-type gene, knockdown of RPL11 reversed the decreased cell viability through activation of the P53 pathway. Altogether, these data demonstrate that RPL11 is closely related to the sensitivity of gastric cancer against 5-FU and activates the P53 pathway, including P21 and Fas, resulting in suppression of tumor progression (Fig. 4).

5-FU is used as an anti-cancer drug for various cancer types, including gastrointestinal cancers, breast cancers, lung cancers, and cancers of the aerodigestive tract (1), indicating that 5-FU may exert a tumor suppression effect via the RPL11-P53 signaling pathway against not only gastric cancers but also other cancer types. It has been reported that 5-FU also induces DNA damage-mediated activation of the P53 pathway (24). In the present study, RPL11 expression was closely related to P53 pathway-mediated cell growth suppression in 5-FU treated gastric cancer, but in other cancer types treated with 5-FU.
Therefore, DNA damage may be predominantly related to P53 pathway-mediated cell growth suppression. Additionally, it has been reported that 5-FU-induced cell growth suppression via inhibition of rRNA processing involves not only RPL11, but also RPL5 and RPL23 in osteosarcoma cells (14), suggesting RPL5 and RPL23 may also be involved in P53 pathway-mediated cell growth suppression in 5-FU treated gastric cancer. Our data indicated that there are some differences in RPL11 expression levels in patients with gastric cancer. RPL11 mRNA expression is regulated by c-Myc and N-Myc, members of the Myc oncoprotein family of transcription factors $(25,26)$. Deferential expression levels and/or activity of Myc in gastric cancer patients may reflect the differences in RPL11 expression levels in these patients. Recently, Wang et al reported that enhancer of zente homologue 2 (EZH2) contributes to 5-FU resistance in gastric cancer and that high $E Z H 2$ expression is correlated with 
A
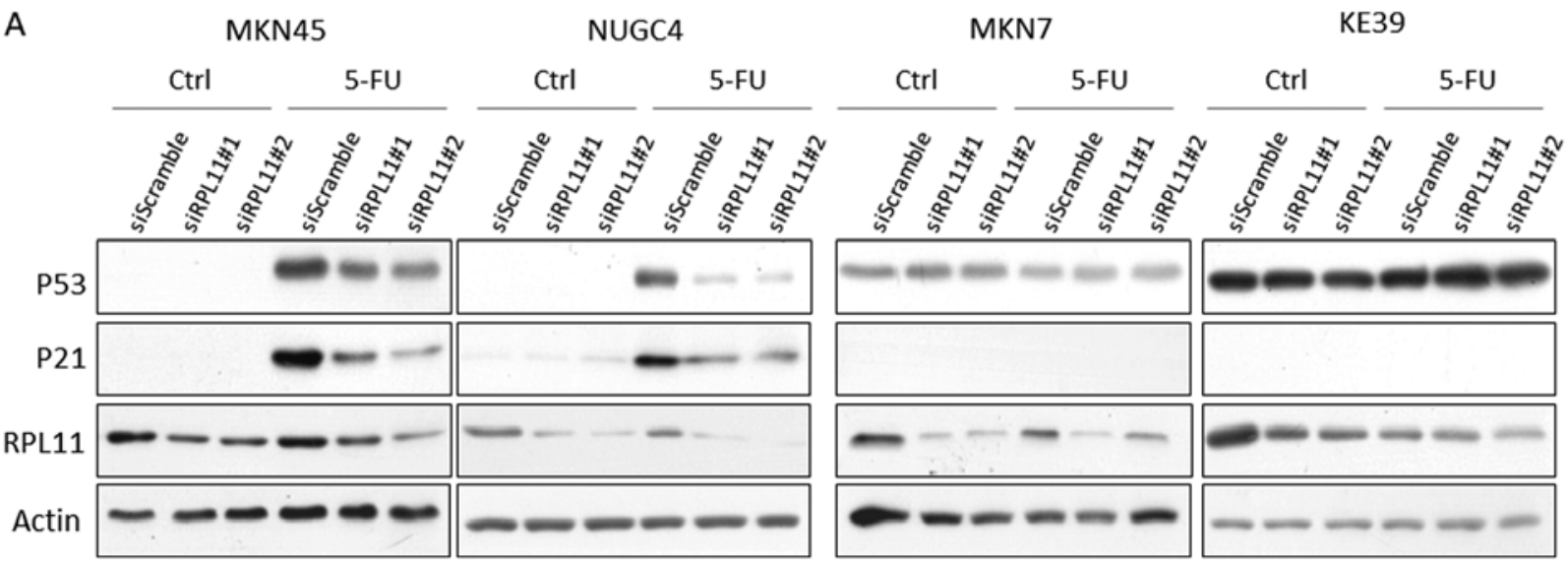

B

P21
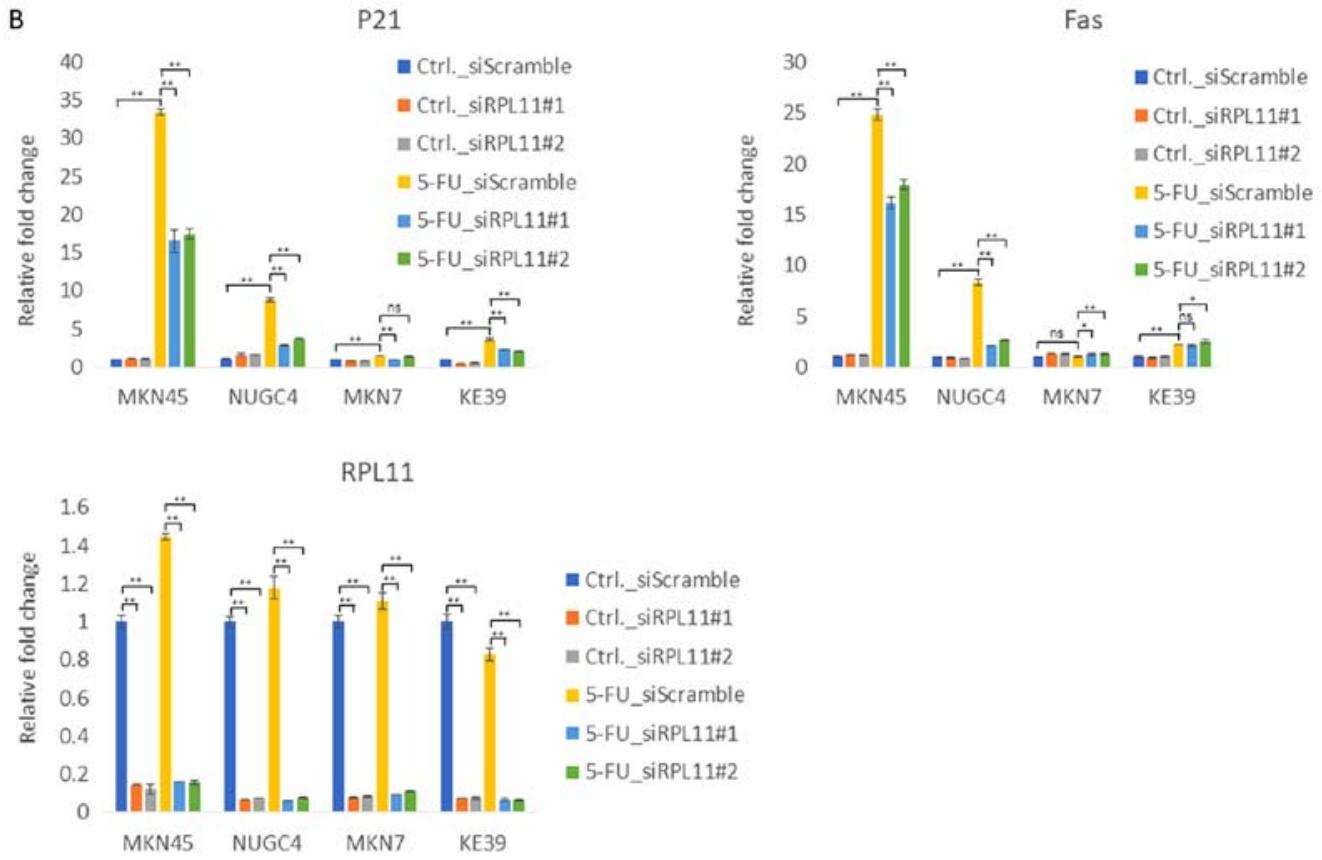

Figure 3. RPL11 is involved in activation of the P53 pathway in 5-FU treated gastric cancer cells. (A) Expression levels of P53, P21 and RPL11 in MKN45, NUGC4, MKN7 and KE39 cells transfected with siScramble (SC), siRPL11\#1, or siRPL11\#2 under mock and 300 $\mu \mathrm{M} 5$-FU treatment for $24 \mathrm{~h}$ were analyzed by immunoblot assay. (B) Relative P21, FAS, and RPL11 expression in MKN45, NUGC4, MKN7, and KE39 cells transfected with siSC, siRPL11\#1, or siRPL11\#2 under mock and $300 \mu \mathrm{M} 5$-FU treatment for $24 \mathrm{~h}$ were analyzed by quantitative real-time PCR. Error bars indicate the standard deviation. ${ }^{*} \mathrm{P}<0.05$ and ${ }^{* * *} \mathrm{P}<0.01$ as indicated. ns, not significant; RPL11, ribosomal protein L11; Ctrl, control; 5-FU, 5-fluorouracil; si, small interfering RNA; FAS, Fas cell surface death receptor.

poor prognosis of gastric cancer patients (27). Although further investigation is needed, gastric cancer patients with both high RPL11 expression and low EZH2 expression may show higher sensitivity against 5-FU treatment than those with either high RPL11 or low EZH2 expression alone, which may help to more accurately predict 5-FU sensitivity in gastric cancer. It has been reported that certain ribosomal proteins, including RPS14, RPL5, RPL22, RPL41, RPS7, RPS15a, RPS24, RPS27, and RPL15 are involved in neoplastic transformation and/or cell migration and/or invasion (28). However, there is no report showing the relationship between ribosomal proteins and drug sensitivity in human cancer so far. Thus, our present study is the first report that the ribosomal protein L11 is associated with drug sensitivity and prognosis in gastric cancer via the P53 pathway.

5-FU is one of the most frequently used first-line treatments for patients with advanced gastric cancer $(29,30)$. However, the 5-year survival rate of patients with gastric

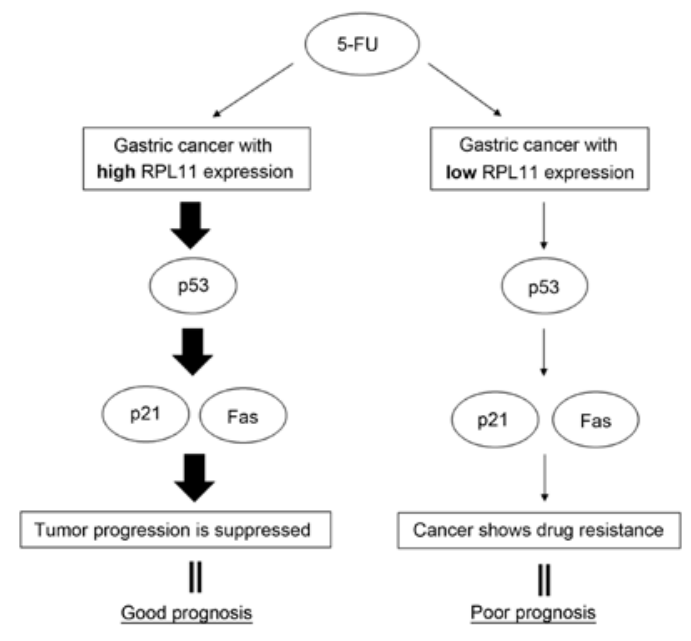

Figure 4. Schematic illustration of relationship between RPL11 expression and sensitivity against 5-FU in gastric cancer. RPL11, ribosomal protein L11; 5-FU, 5-fluorouracil. 
cancer remains poor (31). Therefore, there is an urgent need to identify molecular mechanisms and find novel therapeutic strategies for 5-FU resistance in gastric cancer.

In the present study, we show that RPL11 expression is a crucial factor affecting the sensitivity of gastric cancer against 5-FU, suggesting that RPL11 may be a potential biomarker for predicting 5-FU sensitivity. Identification of RPL11 as a biomarker helps gastric cancer patients with 5-FU resistance avoid unwanted side effects caused by 5-FU treatment. In addition to the role of RPL11 expression as a biomarker, we found RPL11 is functionally required to modulate sensitivity to 5-FU. Therefore, chemotherapy using a drug to elevate RPL11 expression could improve 5-FU resistance in gastric cancer patients with the TP53 wild-type gene. These findings would greatly contribute to a therapeutic strategy for patients with gastric cancer.

\section{Acknowledgements}

The authors would like to thank Ms. Yoshiko Setogawa and Ms. Hiromi Mitsuo (Department of Molecular Oncology, Kagoshima University Graduate School of Medical and Dental Sciences) for their excellent secretarial assistance. We also wish to thank the Joint Research Laboratory, Kagoshima University Graduate School of Medical and Dental Sciences, for use of their facilities.

\section{Funding}

The present study was supported by JSPS KAKENHI (grant nos. JP17J05291, JP15K10311, JP15K10338, JP22501047, JP16K07121, JP17K10871, JP17K07221 and JP18K06732) and the Molecular Profiling Committee, Grant-in-Aid for Scientific Research on Innovative Areas 'Platform of Advanced Animal Model Support' from The Ministry of Education, Culture, Sports, Science and Technology, Japan (JSPS KAKENHI grant no. JP 16H06276), Takeda Medical Foundation, the Shinnihon Foundation of Advanced Medical Treatment Research, Kodama Memorial Fund for Medical Research, the Mochida Memorial Foundation for Medical and Pharmaceutical Research, Foundation for Promotion of Cancer Research in Japan, the Suzuken Memorial Foundation, the Shimabara Science Promotion Foundation and Astellas Foundation for Research on Metabolic Disorders.

\section{Availability of data and materials}

The datasets generated and/or analyzed during the present study are available from the corresponding author on reasonable request.

\section{Authors' contributions}

KK designed the experiments. TK, AS, and MS performed the experiments. TK, AS, MS analyzed the experimental data. TS and KA performed database analysis. KM, MY and YS performed statistical analysis. TK, TH and TF wrote the paper, interpreted data and revised it critically for intellectual content. All authors have read and approved the final manuscript.

\section{Ethics approval and consent to participate}

Not applicable.

\section{Patient consent for publication}

Not applicable.

\section{Competing interests}

The authors declare that they have no competing interests.

\section{References}

1. Longley DB, Harkin DP and Johnston PG: 5-fluorouracil: Mechanisms of action and clinical strategies. Nat Rev Cancer 3: 330-338, 2003.

2. Houghton JA, Tillman DM and Harwood FG: Ratio of 2'-deoxyadenosine-5'-triphosphate/thymidine-5'-triphosphate influences the commitment of human colon carcinoma cells to thymineless death. Clin Cancer Res 1: 723-730, 1995.

3. Sampath D, Rao VA and Plunkett W: Mechanisms of apoptosis induction by nucleoside analogs. Oncogene 22: 9063-9074, 2003.

4. Wilkinson DS and Pitot HC: Inhibition of ribosomal ribonucleic acid maturation in Novikoff hepatoma cells by 5 -fluorouracil and 5-fluorouridine. J Biol Chem 248: 63-68, 1973.

5. Kanamaru R, Kakuta H, Sato T, Ishioka C and Wakui A: The inhibitory effects of 5-fluorouracil on the metabolism of preribosomal and ribosomal RNA in L-1210 cells in vitro. Cancer Chemother Pharmacol 17: 43-46, 1986.

6. Ghoshal K and Jacob ST: Specific inhibition of pre-ribosomal RNA processing in extracts from the lymphosarcoma cells treated with 5-fluorouracil. Cancer Res 54: 632-636, 1994.

7. Bhat KP, Itahana K, Jin A and Zhang Y: Essential role of ribosomal protein L11 in mediating growth inhibition-induced p53 activation. EMBO J 23: 2402-2412, 2004.

8. Dai MS, Zeng SX, Jin Y, Sun XX, David L and Lu H: Ribosomal protein L23 activates p53 by inhibiting MDM2 function in response to ribosomal perturbation but not to translation inhibition. Mol Cell Biol 24: 7654-7668, 2004.

9. Dai MS and Lu H: Inhibition of MDM2-mediated p53 ubiquitination and degradation by ribosomal protein L5. J Biol Chem 279: 44475-44482, 2004.

10. Dai MS, Shi D, Jin Y, Sun XX, Zhang Y, Grossman SR and Lu H: Regulation of the MDM2-p53 pathway by ribosomal protein L11 involves a post-ubiquitination mechanism. J Biol Chem 281: 24304-24313, 2006.

11. Lohrum MA, Ludwig RL, Kubbutat MH, Hanlon M and Vousden KH: Regulation of HDM2 activity by the ribosomal protein L11. Cancer Cell 3: 577-587, 2003.

12. Jin A, Itahana K, O'Keefe K and Zhang Y: Inhibition of HDM2 and activation of $\mathrm{p} 53$ by ribosomal protein L23. Mol Cell Biol 24: 7669-7680, 2004.

13. Zhang Y, Wolf GW, Bhat K, Jin A, Allio T, Burkhart WA and Xiong Y: Ribosomal protein L11 negatively regulates oncoprotein MDM2 and mediates a p53-dependent ribosomal-stress checkpoint pathway. Mol Cell Biol 23: 8902-8912, 2003.

14. Sun XX, Dai MS and Lu H: 5-fluorouracil activation of p53 involves an MDM2-ribosomal protein interaction. J Biol Chem 282: 8052-8059, 2007.

15. Levine AJ and Oren M: The first 30 years of p53: Growing ever more complex. Nat Rev Cancer 9: 749-758, 2009.

16. Nabeya Y, Loganzo F Jr, Maslak P, Lai L, de Oliveira AR, Schwartz GK, Blundell ML, Altorki NK, Kelsen DP and Albino AP: The mutational status of $\mathrm{p} 53$ protein in gastric and esophageal adenocarcinoma cell lines predicts sensitivity to chemotherapeutic agents. Int J Cancer 64: 37-46, 1995.

17. Pickard M, Dive $C$ and Kinsella AR: Differences in resistance to 5 -fluorouracil as a function of cell cycle delay and not apoptosis. Br J Cancer 72: 1389-1396, 1995.

18. Osaki M, Tatebe S, Goto A, Hayashi H, Oshimura M and Ito H: 5-Fluorouracil (5-FU) induced apoptosis in gastric cancer cell lines: Role of the p53 gene. Apoptosis 2: 221-226, 1997. 
19. Wright NA, Poulsom R, Stamp G, Van Noorden S, Sarraf C Elia G, Ahnen D, Jeffery R, Longcroft J, Pike C, et al: Trefoil peptide gene expression in gastrointestinal epithelial cells in inflammatory bowel disease. Gastroenterology 104: 12-20, 1993.

20. Jemal A, Bray F, Center MM, Ferlay J, Ward E and Forman D: Global cancer statistics. CA Cancer J Clin 61: 69-90, 2011

21. Hong CF, Lin SY, Chou YT and Wu CW: MicroRNA-7 compromises p53 Protein-dependent apoptosis by controlling the expression of the chromatin remodeling factor SMARCD1. J Biol Chem 291: 1877-1889, 2016.

22. Yang Y, Nakagawa H, Tetreault MP, Billig J, Victor N, Goyal A, Sepulveda AR and Katz JP: Loss of transcription factor KLF5 in the context of p53 ablation drives invasive progression of human squamous cell cancer. Cancer Res 71: 6475-6484, 2011.

23. Petak I, Tillman DM and Houghton JA: p53 dependence of Fas induction and acute apoptosis in response to 5-fluorouracil-leucovorin in human colon carcinoma cell lines. Clin Cancer Res 6: 4432-4441, 2000

24. Adamsen BL, Kravik KL and De Angelis PM: DNA damage signaling in response to 5-fluorouracil in three colorectal cancer cell lines with different mismatch repair and TP53 status. Int J Oncol 39: 673-682, 2011.

25. Dai MS, Arnold H, Sun XX, Sears R and Lu H: Inhibition of c-Myc activity by ribosomal protein L11. EMBO J 26: 3332-3345, 2007.
26. Boon K, Caron HN, Van Asperen R, Valentijn L, Hermus MC, Van Sluis P, Roobeek I, Weis I, Voûte PA, Schwab M, et al: $\mathrm{N}$-myc enhances the expression of a large set of genes functioning in ribosome biogenesis and protein synthesis. EMBO J 20: 1383-1393, 2001.

27. Wang C, Li X, Zhang J, Ge Z, Chen $\mathrm{H}$ and $\mathrm{Hu}$ J: EZH 2 contributes to 5-FU resistance in gastric cancer by epigenetically suppressing FBXO32 expression. Onco Targets Ther 11: 7853-7864, 2018.

28. Xu X, Xiong X and Sun Y: The role of ribosomal proteins in the regulation of cell proliferation, tumorigenesis, and genomic integrity. Sci China Life Sci 59: 656-672, 2016.

29. Ajani JA: Evolving chemotherapy for advanced gastric cancer. Oncologist 10: 49-58, 2005

30. Zhe W, Feng ZJ and Fang FG: Effect of rhizoma curcumate zedoariae and herbra trifolii repentis on apoptosis of human lung cancer cell line A549. J Capital Univ Med Sci 22: 304-305, 2001.

31. Parkin DM, Bray F, Ferlay J and Pisani P: Global cancer statistics, 2002. CA Cancer J Clin 55: 74-108, 2005.

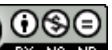

This work is licensed under a Creative Commons Attribution-NonCommercial-NoDerivatives 4.0 International (CC BY-NC-ND 4.0) License. 